\section{Gestão do cuidado integral: estudo de caso em região de saúde da Bahia, Brasil}

\author{
Managing comprehensive care: a case study in a \\ health district in Bahia State, Brazil
}

Adriano Maia dos Santos 1,2 Ligia Giovanella 2

\title{
Resumo
}

Analisou-se a conformação da gestão do cuidado integral nos níveis político-institucional, organizacional e nas práticas, identificando os desafios para constituição de cuidados coordenados entre municípios e os dispositivos para integração e regulação assistencial. Trata-se de estudo de caso, com abordagem qualitativa, na região de saúde de Vitória da Conquista, Bahia, Brasil, que abrange 19 municípios. As fontes de informação foram entrevistas semiestruturadas com gestores, grupos focais com gestores, profissionais e usuários, documentos institucionais e observações. Os resultados indicaram haver insuficiência de médicos para as ações e serviços na Estratégia Saúde da Família, bem como nos serviços especializados, limitando o acesso oportuno. Havia fragmentação entre os diferentes pontos e desarticulação comunicacional na rede. Interesses privados e interferências clientelistas criavam circuitos paralelos, transformando o direito à saúde em troca de favores. Tais questões obstruem as possibilidades de coordenação do cuidado na rede intermunicipal.

Regionalização; Atenção Primária à Saúde; Gestão em Saúde; Integralidade em Saúde; Gestão Clínica

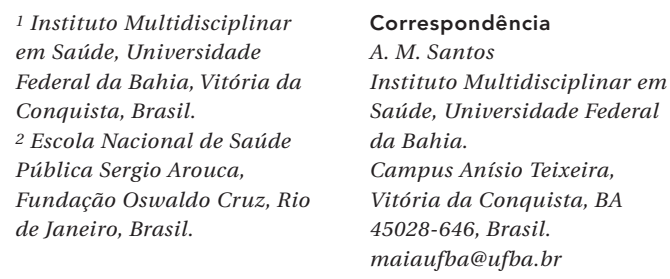

1 Instituto Multidisciplinar em Saúde, Universidade Federal da Bahia, Vitória da Conquista, Brasil.

2 Escola Nacional de Saúde Pública Sergio Arouca, Fundação Oswaldo Cruz, Rio de Janeiro, Brasil.

\author{
Correspondência \\ A. M. Santos \\ Instituto Multidisciplinar em \\ Saúde, Universidade Federal \\ da Bahia. \\ Campus Anísio Teixeira, \\ Vitória da Conquista, $B A$ \\ 45028-646, Brasil.
} maiaufba@ufba.br 


\section{Introdução}

A gestão do cuidado integral 1 está relacionada com a gestão de redes de atenção 2,3 e busca assegurar o princípio da integralidade 4 . Para garantir o cuidado integral à população, gestores precisam responder a um conjunto de problemas de saúde cujo enfrentamento transcende a capacidade de apenas um ente subnacional e, assim, compatibilizar a descentralização políticoadministrativa com a organização regionalizada e hierarquizada de serviços de saúde 5 .

Enquanto em países europeus, com sistemas de saúde universais, as agendas de descentralização e regionalização caminharam articulando-se, nos países latino-americanos, inclusive o Brasil, houve maior destaque para a descentralização, sendo assim, os mecanismos de integração para construção de um sistema regionalizado ficaram em segundo plano 6,7 .

O atraso da regionalização no Sistema Único de Saúde (SUS) pode ter fragilizado a capacidade resolutiva em sistemas locais de saúde ${ }^{8}$, sobretudo na prestação de serviços especializados 9,10, amplificando disputas entre os municípios por recursos 8,11 , ao invés de conformar uma rede de saúde por meio de política territorial compartilhada, criando efeitos paradoxais 7 . A ausência de planejamento regional para as estratégias de descentralização no SUS acirrou as desigualdades dos entes subnacionais, fragilizou e comprometeu o papel das esferas estaduais, resultando, no final da década de 1990, em muitos sistemas locais isolados, especialmente municípios de pequeno porte, sem capacidade de gestão do cuidado integral e sem mecanismos e expertise para conformação de redes intermunicipais 6,12. Ainda assim, estudos sinalizam que a descentralização, apesar dos grandes desafios, logrou êxito em muitos sistemas locais 13,14,15.

A regionalização é um atributo dos sistemas nacionais de saúde que se organizam para ampliar acesso e diminuir desigualdades 16. Porém, há dificuldades na consolidação de regiões de saúde diante da insuficiência no financiamento, frágeis políticas de gestão do trabalho e constrangimentos na oferta de serviços 17 . Além disso, influências do setor privado no SUS 18,19; tensionadas por conflito e disputa entre os municípios “à revelia da discussão regional" 11 fragilizam a gestão do cuidado integral. Em contrapartida, destacam-se o papel estratégico do gestor estadual 20 , as comissões intergestores regionais (CIR) 8,19 e o consórcio intermunicipal de saúde 21 na indução da regionalização. Essas pesquisas revelam, ainda, contribuições da regionalização para a integralidade da atenção, entretanto sua implementação tem sido complexa e incremental, alternando avanços e retrocessos 12,22.

Em sistemas europeus, diferentes estudos 23,24,25 defendem que sistemas integrados devem apoiar-se na atenção primária à saúde como coordenadora para atingir alguns objetivos básicos como cuidado continuado, longitudinalidade, resolubilidade no nível mais adequado, melhora progressiva dos resultados, equidade e uso adequado dos recursos. A coordenação é entendida como uma conexão harmoniosa de diferentes serviços, necessários para prover cuidado continuado das pessoas, articulados para resolver as necessidades dos usuários na sua integralidade 26 .

Em pesquisa recente nesta mesma região de saúde 18 , focou-se a análise na governança regional e foram constatados conflitos e ambiguidades na formulação de políticas locorregionais, pois havia permanente tensionamento entre interesses particulares no âmbito municipal e necessidades regionais de cunho mais solidário.

Extensa revisão sobre integração de sistemas de saúde 27 aponta insuficiência de estudos empíricos que forneçam evidências para tomada de decisão e falta de ferramentas validadas e padronizadas para avaliação dos resultados, o que torna a tarefa de medir e comparar o impacto da integração no nível das práticas, serviços e sistema um grande desafio para gestores e pesquisadores. Nessa perspectiva, preencher parte destas lacunas é uma das intenções desta pesquisa.

Este artigo analisa a conformação da gestão do cuidado integral, na região de saúde de Vitória da Conquista, Bahia, Brasil, nos níveis políticoinstitucional, organizacional e nas práticas, identificando os desafios para constituição de cuidados coordenados entre municípios e os dispositivos para integração e regulação assistencial na região.

\section{Metodologia}

Foi realizado estudo de caso 28 , com abordagem qualitativa, na região de saúde de Vitória da Conquista, abrangendo 19 municípios da Bahia (Tabela 1), entre 2011 e 2012. Com alguma tradição de organização da rede local, Vitória da Conquista apresentava experiências exitosas em distintos estudos 13,29,30, carecendo, entretanto, de investigação para conhecer sua interface para produção da gestão do cuidado integral na região. O Decreto no 7.508 define regiões de saúde como espaços privilegiados para integração dos serviços de saúde e coordenação do cuidado, tendo como ponto de partida o Plano Diretor Regional, favorecendo a ação cooperativa intergestora 31 . 
Municípios, cobertura pela Estratégia Saúde da Família (ESF) e número de equipes de saúde da família e de saúde bucal. Região de Saúde de Vitória da Conquista, Bahia, Brasil, 2012.

\begin{tabular}{|c|c|c|c|c|c|c|c|c|}
\hline Município & $\begin{array}{c}\text { População } \\
2010\end{array}$ & $\begin{array}{c}\text { Gestão da saúde } \\
2012\end{array}$ & $\begin{array}{c}\text { Taxa de } \\
\text { urbanização } \\
2010(\%)\end{array}$ & $\begin{array}{l}\text { IDHM } \\
2010\end{array}$ & $\begin{array}{l}\text { Equipes de saúde } \\
\text { da família } \\
2012\end{array}$ & $\begin{array}{c}\text { Cobertura } \\
\text { pela ESF } \\
2012\end{array}$ & $\begin{array}{c}\text { Equipes de } \\
\text { saúde bucal } \\
2012\end{array}$ & $\begin{array}{l}\text { NASF } \\
(2012)\end{array}$ \\
\hline Anagé & 25.516 & Estadual & 19,4 & 0,540 & 7 & 82,64 & 2 & 1 \\
\hline Barra do Choça & 34.788 & Municipal & 49,2 & 0,551 & 12 & 100,00 & 4 & - \\
\hline Belo Campo & 16.021 & Municipal & 56,2 & 0,575 & 6 & 100,00 & 2 & - \\
\hline Bom Jesus da Serra & 10.113 & Estadual & 27,6 & 0,546 & 3 & 100,00 & 3 & - \\
\hline Caetanos & 13.639 & Estadual & 23,6 & 0,542 & 4 & 100,00 & 3 & - \\
\hline Cândido Sales & 27.918 & Estadual & 69,2 & 0,601 & 6 & 62,17 & 3 & - \\
\hline Caraíbas & 10.222 & Estadual & 24,6 & 0,555 & 4 & 100,00 & 3 & - \\
\hline Condeúba & 16.898 & Estadual & 44,5 & 0,582 & 6 & 100,00 & 5 & - \\
\hline Cordeiros & 8.168 & Estadual & 31,2 & 0,579 & 4 & 100,00 & 2 & - \\
\hline Encruzilhada & 23.766 & Estadual & 21,7 & 0,544 & 4 & 57,58 & 4 & - \\
\hline Maetinga & 7.038 & Municipal & 40,6 & 0,538 & 4 & 100,00 & 2 & 1 \\
\hline Mirante & 10.507 & Estadual & 17,3 & 0,527 & 4 & 100,00 & 4 & - \\
\hline Piripá & 12.783 & Estadual & 48,7 & 0,575 & 6 & 100,00 & 2 & - \\
\hline Planalto & 24.481 & Estadual & 61,0 & 0,560 & 6 & 83,83 & 3 & 1 \\
\hline Poções & 44.701 & Estadual & 77,0 & 0,604 & 10 & 77,14 & 8 & 1 \\
\hline Pres. Jânio Quadros & 13.652 & Municipal & 31,0 & 0,542 & 7 & 100,00 & 3 & 1 \\
\hline Ribeirão Largo & 8.602 & Estadual & 53,1 & 0,540 & 4 & 100,00 & 2 & - \\
\hline Tremedal & 17.029 & Estadual & 23,1 & 0,528 & 5 & 100,00 & 3 & 1 \\
\hline Vitória da Conquista & 306.866 & Municipal & 89,6 & 0,678 & 37 & 41,16 & 29 & 4 \\
\hline Região de Saúde & 632.708 & - & - & - & 139 & 65,00 & 87 & 10 \\
\hline Bahia & 14.016 .906 & - & 72,1 & 0,660 & 2.748 & 62,80 & 1.911 & 116 \\
\hline
\end{tabular}

IDHM: índice de desenvolvimento humano municipal; NASF: Núcleo de Apoio à Saúde da Família.

Nota: competência dezembro de 2011.

Fonte: Departamento de Atenção Básica, Secretaria de Atenção à Saúde, Ministério da Saúde/Coordenação de Avaliação e Monitoramento, Diretoria de Atenção Básica, Secretaria da Saúde do Estado da Bahia.

Este estudo apoiou-se em três dimensões que compõem a gestão do cuidado ${ }^{1}$ : profissional, organizacional e sistêmica. A dimensão profissional refere-se ao processo de trabalho na esfera do encontro entre usuário e profissional. A dimensão organizacional corresponde ao componente institucional das práticas, referindo-se ao nível das relações entre diferentes profissionais e tecnologias disponíveis para operacionalizar o cuidado. A dimensão sistêmica engloba as anteriores e representa a modelagem da rede de cuidados que se dá num determinado território (municipal ou regional), imprescindível para garantia da integralidade.

A gestão do cuidado integral superpõe-se com noções de integralidade. Nesta pesquisa, a integralidade remete à integração de serviços em redes locorregionais, requer interdependência/ cooperação entre sujeitos/instituições, compartilhando projetos terapêuticos (coordenação), conformando um "sistema sem muros" 4.
Foi construída uma "matriz para análise da gestão do cuidado integral em rede regionalizada”, por meio da releitura das dimensões da gestão do cuidado ${ }^{1}$, em três níveis: político-institucional, organizacional e práticas. O nível político-institucional responde pelo arcabouço legal, pelas propostas/intencionalidades e ações dos sujeitos para implementação de redes regionais. O nível organizacional abarca uma dimensão operativa, que se dá nas interfaces entre diferentes estabelecimentos e organizações nas regiões de saúde, sofrendo intervenções e interações dos diferentes sujeitos que constituem e imprimem uma lógica para gestão e produção na rede. O nível das práticas compreende as relações dos profissionais entre si e com usuários.

Ressalta-se que os três níveis apresentam dificuldades teóricas para definição das fronteiras entre si e, portanto, carreiam certa arbitrariedade na sua delimitação. Para validação, o conteúdo da matriz foi submetido, por correio eletrônico, a 
um comitê de especialistas, contando com a colaboração de cinco pesquisadores de instituições distintas. Os critérios de seleção foram: ter experiência, pesquisar e publicar em avaliação de serviços de saúde, ter conhecimento metodológico pertinente ao tema. Não há consenso quanto ao número ideal de especialistas 32 e seguiu-se recomendação de Lynn 33 .

Os resultados emergiram das análises e cruzamentos de dados coletados em 17 entrevistas semiestruturadas com gestores/gerentes (esferas estadual, regional e municipal), nove grupos focais - quatro grupos com profissionais (GF-P), compostos por médicos e enfermeiros em cada, quatro grupos com usuários (GF-U) e um grupo com gestores (GF-G) -, registros de observações na CIR (sete reuniões entre outubro de 2011 a março de 2012), em unidades de saúde da família (USF) e centrais de marcação de consultas (CMC), complementados por documentos: atas da CIR (2011-2012) e relatórios anuais de gestão (RAG) de 2011.

Para análise final, fez-se entrecruzamento entre diferentes informações coletadas, complementado-as com os documentos, identificandose convergências e divergências. As análises seguiram critérios/indicadores pré-definidos da matriz para cada nível, elegendo-se duas dimensões que conformaram os resultados e discussão: a) Coordenação do cuidado na região de saúde (Tabela 2): trata-se de um atributo da atenção primária à saúde e funciona como amálgama, pois potencializa e viabiliza cuidados continuados, articula os diferentes pontos no sistema para a integralidade e parte do reconhecimento que diferentes necessidades das pessoas e possibilidades de resolução não podem ser alcançadas apenas pela equipe de atenção primária à saúde 34; b) Dispositivos para integração e regulação assistencial na região de saúde (Tabela 3): a integração dos serviços é a contraposição aos modelos fragmentados. Serviços de saúde integrados em forma de redes de atenção tendem a atuar de maneira equilibrada sobre as condições agudas e crônicas, desde que estejam organizados por um conjunto coordenado de pontos de atenção à saúde 3 .

A pesquisa foi aprovada pelo Comitê de Ética em Pesquisa da Escola Nacional de Saúde Pública Sergio Arouca, Fundação Oswaldo Cruz, em 4 de outubro de 2011, parecer no 207/ 11 .

\section{Resultados e discussão}

\section{Coordenação do cuidado na região de saúde}

Os principais resultados sobre coordenação do cuidado, nos três níveis, foram sintetizados na Tabela 4. Em suma, a coordenação entre municípios era incipiente, posto que os resultados, nas diferentes fontes de informação, detectaram que mecanismos de compartilhamento de informações clínicas, fundamentais para a continuidade do cuidado, resumiam-se às guias de encaminhamento, aos laudos de exames e ao relato verbal do próprio usuário, após consulta com especialista. Além disso, convivia-se com um modelo médico-centrado e, paradoxalmente, muitas USF "sem médico" ou médicos restritos a poucos turnos semanais.

As entrevistas com gestores foram convergentes com grupos focais com profissionais ao apontarem que na Estratégia Saúde da Família (ESF) especialmente médicos eram responsáveis pelas referências, por meio de guias de solicitação, mediados por centrais de marcação de consultas que restringiam os encaminhamentos às regras administrativas sem possibilidade de escolha entre os prestadores. Nesse aspecto, as práticas na ESF parecem não diferir das práticas dominantes na rede básica tradicional apontadas em outros estudos avaliativos 35,36,37. Ainda assim, não foram identificadas nas entrevistas e observações na CIR ações voltadas a tornar a ESF coordenadora do cuidado.

Gestores afirmaram que médicos agiam como gatekeeper no sentido da obrigatoriedade de sua referência para que usuários acessassem outros pontos da rede, entretanto, sem lograr mecanismos articulados entre diferentes níveis, ou seja, sem continuidade e sem coordenação, limitados à função burocrática e constrangidos pela insuficiência de vagas/cotas. No fragmento, profissionais apontam para um acesso racionado pela oferta: “... a demanda de ultrassonografia é muito grande e o quantitativo de vagas oferecido é muito pouco. Tem que ficar selecionando" (GF-P3).

Assim, os mais prejudicados eram usuários que procuravam a porta mais acessível para expor sua demanda, em momentos de grande fragilidade, ficando vulneráveis e propensos a serem manipulados, revertendo um direito num suposto favor. Essa evidência convergiu entre gestores, profissionais e usuários. De forma emblemática, usuários expõem a manipulação política na oferta de vagas: “...tem uma pessoa na cidade que é vista por muitos pacientes como uma deusa. Diz ela, que é porque tem muito conhecimento com os médicos. E as pessoas iludidas acabam deixando de buscar o lugar correto. E não só existe ela, exis- 
Matriz para análise da gestão do cuidado integral em rede regionalizada de saúde - Coordenação do cuidado. Região de Saúde de Vitória da Conquista, Bahia, Brasil, 2012.

\begin{tabular}{|c|c|c|}
\hline $\begin{array}{l}\text { Nível político-institucional } \\
\text { Critérios/Indicadores }\end{array}$ & $\begin{array}{l}\text { Nível organizacional } \\
\text { Critérios/Indicadores }\end{array}$ & $\begin{array}{l}\text { Nível das práticas } \\
\text { Critérios/Indicadores }\end{array}$ \\
\hline $\begin{array}{l}\text { - Estratégia de incentivo e fomento para expansão } \\
\text { da cobertura e adequação da ESF na região; } \\
\text { - Estratégia para adequação da estrutura física e } \\
\text { gerencial das USF nos municípios da região; } \\
\text { - Estratégias para ampliação do escopo da ESF; } \\
\text { - Estratégias para que a referência via equipes } \\
\text { de saúde da família seja requisito para acessar os } \\
\text { demais níveis; } \\
\text { - Estratégia para que o usuário seja acompanhado } \\
\text { pela equipes de saúde da família em todos os níveis } \\
\text { e permaneça ao longo do tempo. }\end{array}$ & $\begin{array}{l}\text { • Existência e aplicação de metas } \\
\text { regionais de expansão da cobertura e } \\
\text { fortalecimento da ESF; } \\
\text { - Existência de adequação das USF para } \\
\text { as ações da equipes de saúde da família } \\
\text { na região; } \\
\text { • Conectividade nas USF; } \\
\text { • Incentivo financeiro à gerência } \\
\text { compartilhada USF; } \\
\text { - Municípios que aderiram ao PMAQ; } \\
\text { • Existência/tipo de incentivo para } \\
\text { incorporação de apoio às equipes de } \\
\text { saúde da família; } \\
\text { • Incorporação de equipamentos } \\
\text { e insumos à USF para aumento de } \\
\text { adensamento tecnológico da ESF; } \\
\text { • Existência de incentivo ao trabalho } \\
\text { multiprofissional e apoio institucional; } \\
\text { - Mecanismo de regulação que atrela } \\
\text { à equipes de saúde da família os } \\
\text { encaminhamentos para as consultas } \\
\text { existência de para valorização do papel de } \\
\text { coordenação/longitudinalidade da } \\
\text { equipes de saúde da família. }\end{array}$ & 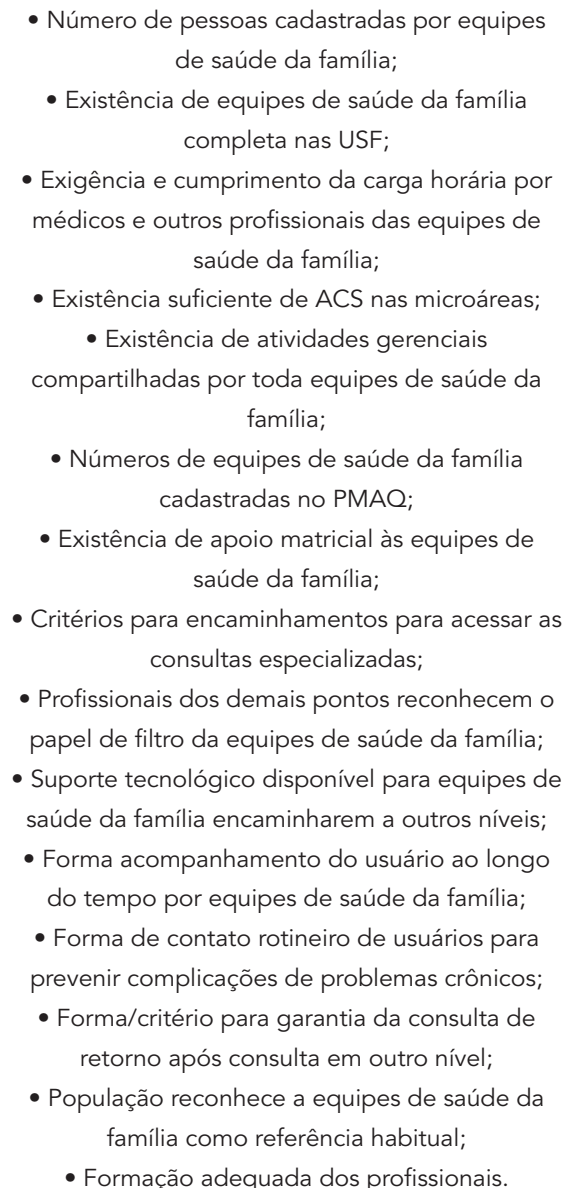 \\
\hline
\end{tabular}

ACS: agentes comunitários de saúde; ESF: Estratégia Saúde da Família; PMAQ: Programa Nacional de Melhoria do Acesso e da Qualidade da Atenção Básica; USF: unidades de saúde da família.

tem outras pessoas também que trabalham em cima da politicagem" (GF-U3).

Em contraste, em países europeus organizados por sistemas nacionais de saúde é comum que a porta de entrada seja uma unidade de atenção primária à saúde, que utiliza a prerrogativa de filtro (gatekeeper) para encaminhamento dos usuários aos demais pontos da rede, facilitando, assim, a coordenação por um médico generalista 38 .

Observações na CIR e USF, relatórios de gestão, entrevistas com gestores e grupos focais convergiram na perspectiva de reconhecer a insuficiência de médicos para as ações e serviços na ESF, notadamente nos menores municípios da região e unidades da zona rural. De forma ilustrativa, um gestor municipal fala sobre a adesão médica condicionada ao salário “...não se acha profissional médico. E o valor é lá em cima, o município não tem condições de arcar com o médico para ficar de 2a a $6 \underline{a}$ " (Ent.3-GM). A dificuldade de atração e fixação de médicos, atrelada à ausência de política consistente de gestão do trabalho abria espaço para acordos informais, entre gestores e profissionais, que "autorizam" redução na carga horária, flexibilização das funções a serem desenvolvidas, pagamento de salários acima da média, colaborando para aumentar a rotatividade de profissionais e competição entre municípios. Tal perspectiva é sinalizada, também, por 
Matriz para análise da gestão do cuidado integral em rede regionalizada de saúde - Integração e regulação assistencial. Região de Saúde de Vitória da Conquista, Bahia, Brasil, 2012.

\begin{tabular}{|c|c|c|}
\hline $\begin{array}{l}\text { Nível político-institucional } \\
\text { Critérios/Indicadores }\end{array}$ & $\begin{array}{l}\text { Nível organizacional } \\
\text { Critérios/Indicadores }\end{array}$ & $\begin{array}{l}\text { Nível das práticas } \\
\text { Critérios/Indicadores }\end{array}$ \\
\hline $\begin{array}{l}\text { - Estratégia para oferta de serviços, buscando } \\
\text { superar os vazios assistenciais; } \\
\text { - Estratégia para fortalecer a rede própria (pública) } \\
\text { de serviços especializados; } \\
\text { - Estratégias para implantação e qualificação de } \\
\text { central de regulação e marcação de consultas e } \\
\text { exames especializados na região; } \\
\text { - Estratégias para implantação e qualificação de } \\
\text { central de regulação e controle de leitos; } \\
\text { - Estratégias para implantação, qualificação e uso } \\
\text { de protocolos clínicos, prontuário eletrônico; } \\
\text { - Estratégia de monitoramento das filas de espera } \\
\text { para atenção especializada; } \\
\text { - Estratégia para distribuição e/ou oferta adequada } \\
\text { de SADT. }\end{array}$ & 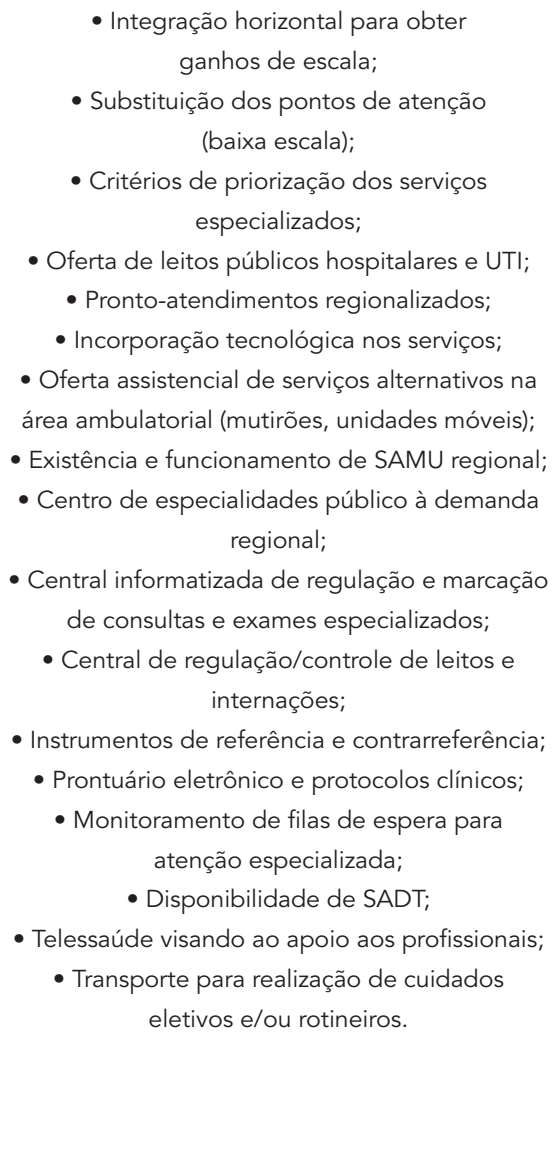 & 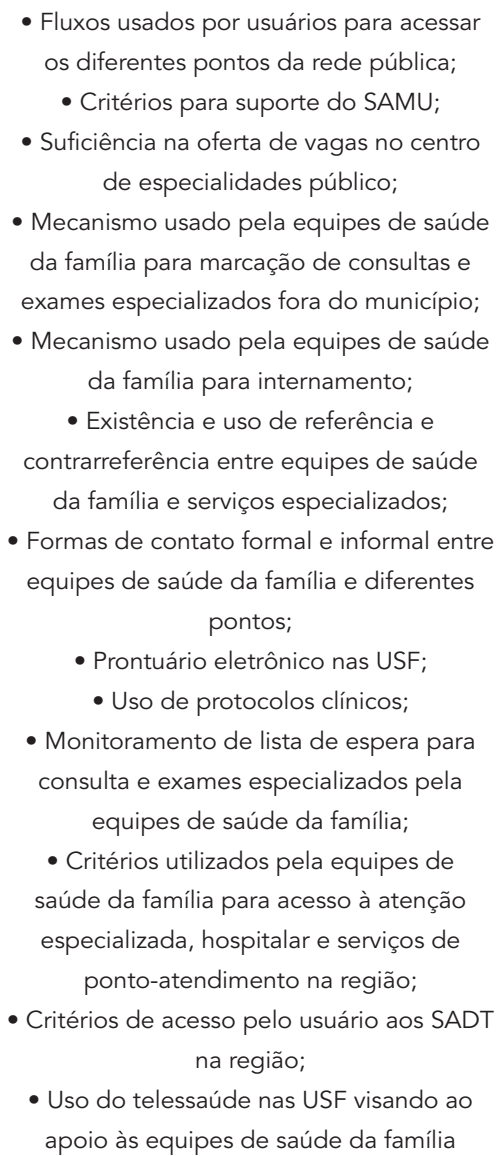 \\
\hline
\end{tabular}

SADT: Serviço Auxiliar de Diagnóstico e Terapia; SAMU: Serviço de Atendimento Móvel de Urgência; USF: unidades de saúde da família; UTI: unidade de tratamento intensivo.

profissionais: "nós [médicos] temos um salário bom no interior, um salário que a gente não tem na cidade maior. Conseguimos trabalhar uma carga horária menor. Eu trabalho três dias na semana..." (GF-P2).

A atração e fixação de médicos, para atuarem na atenção primária à saúde, particularmente em zonas remotas e desfavorecidas, não é um problema exclusivo do Brasil, ao contrário, é um desafio na maioria dos países americanos 39 e europeus 40,41 . Na região estudada, superpõem-se três questões críticas da gestão do trabalho médico no SUS: insuficiência de médicos em relação ao total da população, distribuição desigual nas diferentes regiões (grandes centros, pequenos municípios e zonas rurais) e inadequação na formação médica para atuação em atenção primária à saúde. Um grupo focal ressalta a problemática da formação “...quem devia trabalhar em ESF é quem tinha formação em ESF. Mas (...) todo mundo que se forma, o primeiro galho que pega é ESF. Isso, enquanto não forma e faz residência" (GF-P4).

Na região, conforme atas da CIR e entrevistas com gestores, não havia exigência de formação específica, nem acúmulo de experiências para trabalhar na ESF. Além disso, os dados apontam como justificativas para não permanência e rotatividade de médicos, as condições de trabalho, carga horária considerada elevada, salários percebidos como incompatíveis com as funções as ESF, sobrecarga de trabalho, dificuldade para 
Síntese de resultados - Coordenação do cuidado. Região de Saúde de Vitória da Conquista, Bahia, Brasil, 2012.

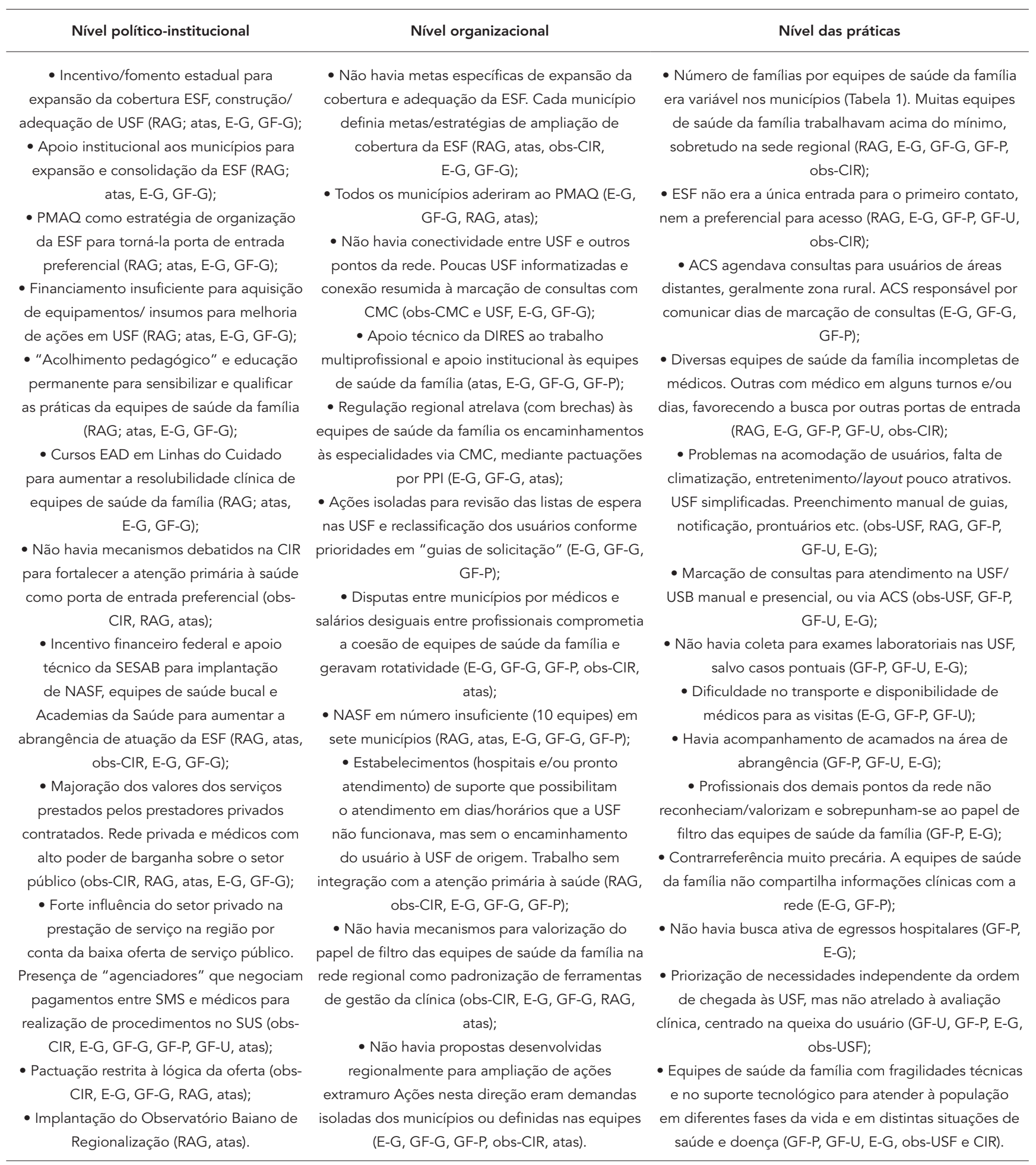

ACS: agentes comunitários de saúde; CIR: comissões intergestores regionais; CMC: centrais de marcação de consultas; DIRES: Diretoria Regional de Saúde; EAD: educação à distância; ESF: Estratégia Saúde da Família; E-G: entrevista semiestruturada com gestor (estadual, municipal e regional); GF-G: grupo focal com gestor estadual; GF-P: grupo focal com profissionais; GF-U: grupo focal com usuários; NASF: Núcleo de Apoio à Saúde da Família; obs: registro de observações; PMAQ: Programa Nacional de Melhoria do Acesso e da Qualidade da Atenção Básica; PPI: programação pactuada e integrada; RAG: Relatório Anual de Gestão; SESAB: Secretaria da Saúde do Estado da Bahia; SMS: secretarias municipais de saúde; SUS: Sistema Único de Saúde; USB: unidades de saúde bucal; USF: unidades de saúde da família. 
obter suporte de especialista, pouca identificação com medicina da família, além do vínculo de trabalho precário e certo isolamento dos pares. Tais achados são convergentes com outros estudos 42,43 . No Canadá, por exemplo, a satisfação entre médicos de família tem importante implicação para sustentabilidade e eficiência dos serviços, bem como é determinante na retenção ou rotatividade dos médicos, podendo afetar no desempenho dos mesmos e no interesse de jovens médicos optarem pela carreira 44 .

As USF, segundo relatórios de gestão e observações nos municípios, localizavam-se preferencialmente em zonas periféricas e rurais, por conseguinte abarcavam uma população de menor renda e com um conjunto de necessidades sociais básicas (saneamento, habitação, educação etc.) não atendidas pelo poder público, exigindo competência dos profissionais para além da assistência à saúde stricto sensu. Ainda assim, mesmo quando localizadas em territórios com pessoas de diferentes estratos sociais em sua área de abrangência, as USF são acessadas de maneira seletiva e focalizada 45 , de tal forma que a busca é maior em populações com menor nível socioeconômico e sem cobertura por seguro privado 46 .

Além da restrição de médicos, horários de funcionamento das USF contribuíam para a dificuldade de acesso de grupos que trabalhavam, estudavam ou exerciam atividade em horário comercial. A adscrição por área de residência torna-se um entrave a pessoas que cumprem alguma atividade laboral em áreas distantes da USF de referência, requerendo dos gestores e profissionais plasticidade na organização da oferta. Usuários sintetizaram tal realidade: “...se tiver com consulta marcada menos mal, mas se adoecer de repente e chegar lá [USF], de repente, ser atendido, não é fácil não. (...) a gente acaba ficando em casa com chazinhos. É que tem muita gente que tem prioridade. Eu até evito. Eu só venho [na USF] no último caso" (GF-U4). Esses aspectos fragilizam a USF como lócus de primeiro contato e busca regular, por contribuir para que usuários procurem outros serviços, particularmente o de pronto-atendimento, mesmo nos casos de problemas que poderiam ser resolvidos no âmbito da atenção primária à saúde.

Essa miríade de fatores objetivos e subjetivos repercute nas escolhas dos usuários e disposição para elegerem a USF como prioridade na rede.

\section{Dispositivos para integração e regulação assistencial na região de saúde}

Os principais resultados sobre integração, nos três níveis, foram sintetizados na Tabela 5. As diferentes fontes indicam que a integração es- barrava no subfinanciamento público com restrição de serviços especializados. De acordo com observações, atas da CIR e gestores, constatou-se que a população que necessitava de assistência convivia com problemas de acesso que comprometiam a continuidade da atenção em decorrência, também, da fragmentação entre diferentes pontos e desarticulação com a ESF.

Esse mosaico de problemas parece partilhar de gênese comum, sendo consequência da indefinição de um modelo de atenção coerente com a gestão do cuidado em todos os níveis. Um grupo de usuários alertou para o tempo de espera para exames especializados: “o doutor me pediu ultrassom da mama e eu não conseguia fazer. Porque eu vim [na USF] várias vezes para marcar e o rapaz falava: 'Hoje está fora do sistema, hoje não tem vaga, vem dia 20'. Eu vim três meses seguidos, mas todo dia 20 eu vinha e não conseguia. Desisti!' (GF-U3). Especificamente em relação ao exame de ultrassonografia mamária, a dificuldade no acesso decorria da insuficiência na oferta do serviço na região.

Segundo gestores, a programação pactuada e integrada (PPI) mostrava-se com limites decorrentes na defasagem do financiamento, mas notadamente por não lograr reverter questões de vazios assistenciais e reproduzir os problemas já cristalizados na rede. Apesar disso, a PPI é uma ferramenta importante na racionalização dos recursos financeiros e alocação territorial dos serviços conforme escala e escopo 47 , todavia não estava atrelada a planejamento locorregional que buscasse transformar o núcleo tecnológico procedimento-centrado numa matriz tecnológica que incidisse na forma de produção do cuidado nos territórios e captasse as necessidades da população. Tais questões estão sintetizadas pelo gestor municipal: "PPI é questão de dividir o bolo para muita gente, sem ter como todo mundo pegar uma fatia (...). PPI não tem aumento de recurso, faz uma distribuição, aloca recurso de oferta dentro da região, um recurso já existente, que aí redistribui, tirando de um e colocando para outro" (Ent.7-GM).

Em contraste, há dificuldades de referenciamento no SUS mesmo nas regiões ricas, com diversidade de prestadores e farta oferta de serviços de média e alta densidade 48 . Esses achados corroboram a necessidade de mudança no núcleo tecnológico do cuidado que modelou os níveis político, organizacional e das práticas na região e que revelou certa inércia dos gestores perante problemas locorregionais, como se eles não fossem passíveis de superação. Tal perspectiva fica marcada no fragmento de um gestor estadual: “Termo de Compromisso de Gestão, (...) no que de fato isso implicou para o gestor municipal? 
Síntese de resultados - Integração e regulação assistencial. Região de Saúde de Vitória da Conquista, Bahia, Brasil, 2012.

\begin{tabular}{|c|c|c|}
\hline Nível político-institucional & Nível organizacional & Nível das práticas \\
\hline 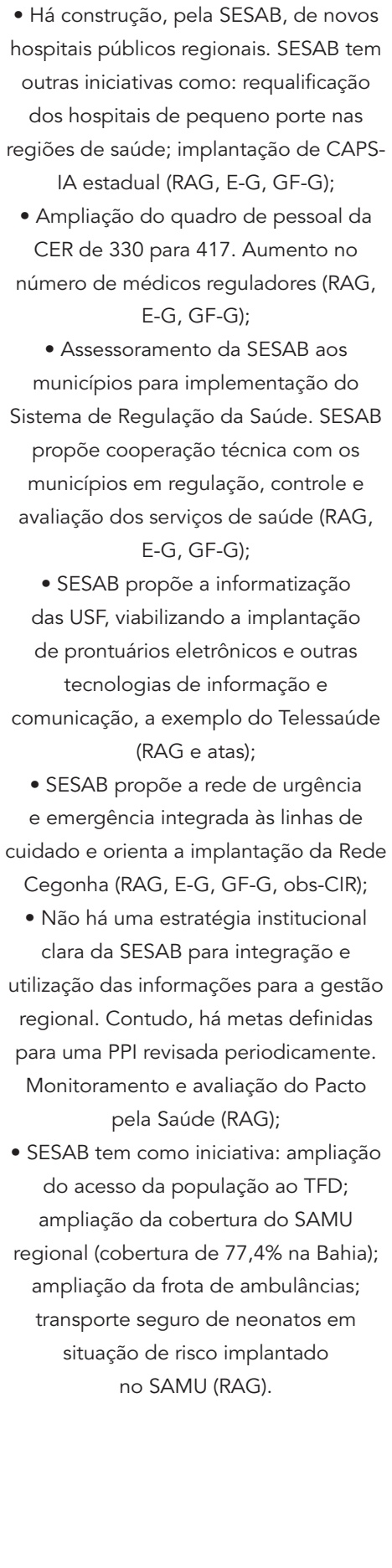 & 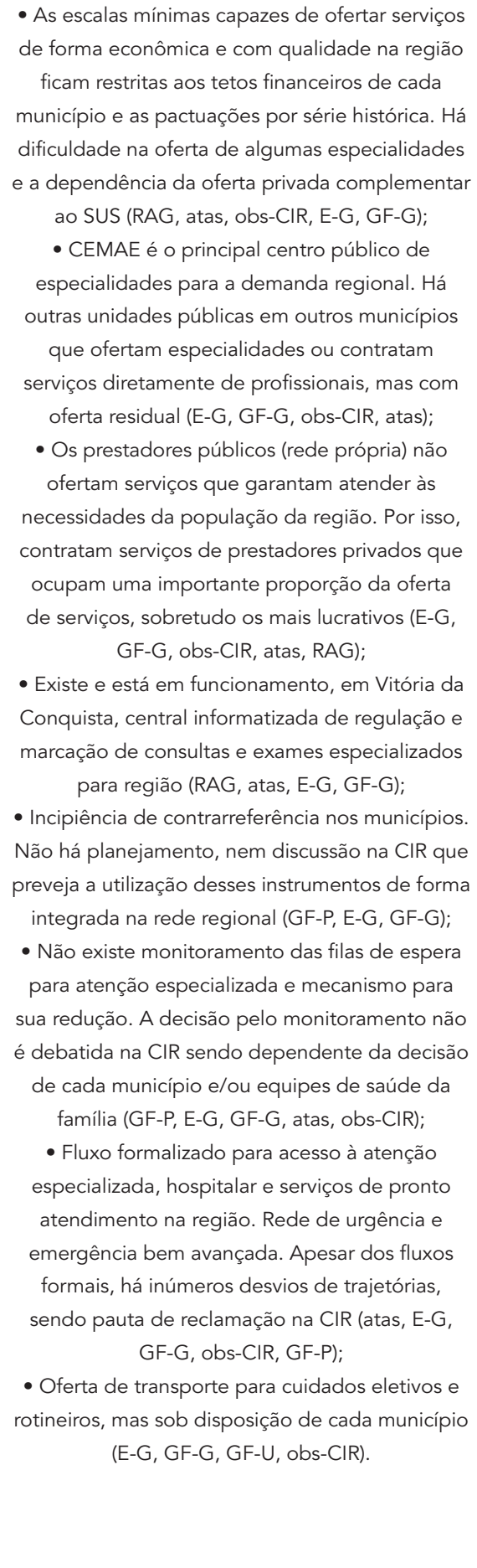 & 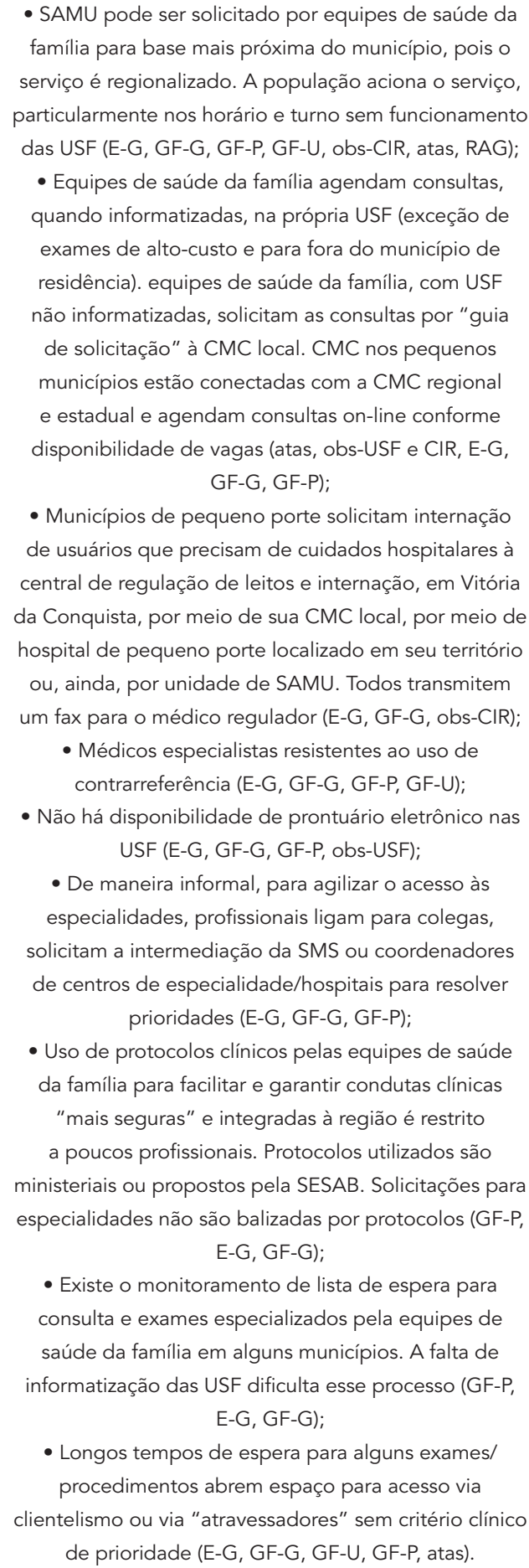 \\
\hline
\end{tabular}

CAPS-IA: Centros de Atenção Psicossocial para Infância e Adolescência; CEMAE: Centro Municipal de Atendimento Especializado; CER: Central Estadual de Regulação; CIR: comissões intergestores regionais; CMC: centrais de marcação de consultas; E-G: entrevista semiestruturada com gestor (estadual, municipal e regional); GF-G: grupo focal com gestor estadual; GF-P: grupo focal com profissionais; GF-U: grupo focal com usuários; obs: registro de observações; PPI: programação pactuada e integrada; RAG: Relatório Anual de Gestão; SAMU: Serviço de Atendimento Móvel de Urgência; SESAB: Secretaria da Saúde do Estado da Bahia; SMS: secretarias municipais de saúde; SUS: Sistema Único de Saúde; USF: unidades de saúde da família; TFD: tratamento fora de domicílio. 
Não enxergo absolutamente nada! Ficou cartorial e nada avançou. (...) garantiu a construção desse espaço [CIR], instância colegiada e representativa (...), mas não necessariamente com corresponsabilidade" (Ent.15-GE).

Para gestores e observações na CIR, o subfinanciado e a não regulação da formação dos profissionais e de suas especialidades conforme necessidade para oferta pública desdobravamse em baixa oferta em serviços de média e alta densidade tecnológica, dificuldades na disponibilidade de médicos e algumas especialidades. A restrição de profissionais e determinados serviços especializados reverberam numa supervalorização financeira e simbólica, tornandoos produtos com elevado "valor de troca". Para ofertá-los, presos unicamente à PPI, os gestores comprometiam os tetos financeiros, não conseguiam atender a demanda que, por sua vez, tende a aumentar, ou seja, permanecem num círculo vicioso, pois retroalimentam as iniquidades.

Além disso, abre-se espaço para importante mercado da saúde em estreita simbiose com o sistema público 48,49 . Somado a isso, os problemas da ESF, discutidos na seção anterior, não apontam para desconstrução dessa lógica produtora de demandas. Ao invés disso, por um lado as equipes tinham dificuldades na retaguarda assistencial, limitando a continuidade e a resolubilidade dos casos, com consequente prolongamento e, até, agravamento dos problemas. Em síntese, ao lado dos casos novos, havia um estoque de problemas que não se resolviam e que, ao mesmo tempo, retornavam ao ponto de partida, vale dizer, novas demandas por serviços especializados e oferta insuficiente para atendê-las, modelando uma espécie de espiral de necessidades.

Em relatórios e atas da CIR, encontrava-se um conjunto de serviços integrados numa rede formal e com base territorial definida, mas que na prática funcionavam de maneira fragmentada e sobreposta, com fluxos comunicacionais incipientes que não viabilizam a coordenação do cuidado e, por conseguinte, a continuidade da atenção. Para gestores, nos pequenos municípios a integração mostrava-se mais frágil, visto que eles, na maior parte das vezes, eram apenas o financiador via PPI, ficando as funções de prestador e regulador nas mãos dos municípios de referência. Além disso, apenas quatro municípios assumiam o comando único e geriam os recursos para a média densidade. O trecho selecionado sintetiza a fala de um gestor regional: “...municípios assumiram, outros não [comando único]. Não assumiram por vontade própria, em alguns casos por orientação da própria coordenação estadual, porque são municípios, ainda, muito inci- pientes na gestão (...) por falta de preparo de quem está no poder" (Ent.13-GR).

Para forjar uma rede que atendesse às necessidades assistenciais, num contexto de insuficiência na oferta, constatou-se a existência de centrais de regulação e marcação de consultas de âmbito regional (sede da região) e local (distintos municípios) que operavam, segundo gestores e observação na CIR, restringindo a oferta aos tetos pré-acordados. Tal racionalidade visava partilhar a oferta segundo poder de compra de cada município. Portanto, a oferta não tinha relação estreita com o perfil epidemiológico e com a necessidade de fato. Apesar disso, estudos indicam que centrais de regulação e marcação de consultas representam importante avanço enquanto instrumento de integração, pois permitem acesso regulado e fluxos formais aos serviços especializados 50 .

Na região, gestores e profissionais concordaram que o processo de regulação/priorização das consultas iniciava-se nas USF, mesmo não informatizadas, em seguida nas CMC locais e, por fim, na CMC regional, mediante avaliação de médico regulador. Tal descentralização permitia um olhar atento às necessidades mais prementes e a diminuição do tempo de espera aos casos mais urgentes/prioritários. Segundo profissionais, a insuficiência na oferta de serviços implicava uma "priorização das prioridades" gerando conflitos éticos. Além do que, para gestores, profissionais e usuários, abria-se espaço para mecanismos informais entre médicos, práticas clientelistas, ou forçava o próprio usuário a buscar alternativas, promovendo iniquidades. O clientelismo, segundo gestor municipal, era uma marca: "interferência política num município pequeno é bastante presente. Às vezes, é um vereador que chega: 'Ah! dá um jeitinho aí.' É claro que as meninas [CMC] vão ter que dar um jeitinho" (Ent.4-GM).

Observou-se, ainda, a fragilidade das CMC nos pequenos municípios, nas quais o técnicomarcador (sem formação específica em saúde) fazia os agendamentos conforme descrição médica nas guias e, paralelamente, assumia importante papel discricionário na priorização de vagas. Ressalta-se que, segundo gestores e profissionais, a participação das equipes de saúde da família na regulação era coadjuvante, uma vez que a priorização era fruto de contingência na oferta que, aliada à incipiência de instrumentos para continuidade informacional não contemplava a coordenação entre níveis. A ausência de instrumentos comunicacionais entre níveis, bem como a fragilidade na organização do trabalho das equipes de saúde da família, sobretudo de médicos, comprometem o atributo da coordenação e favorece a inadequação de diagnósti- 
cos e tratamentos com desdobramentos graves à segurança dos usuários, tanto quanto produz uma sensação de desconfiança dos usuários e profissionais, por conta de processos de trabalhos desarticulados, barreiras administrativas que geram perda de tempo e comprometem a continuidade 51 .

Na região analisada, observou-se a ausência de prontuários eletrônicos nas USF e centros de especialidades, falta de conectividade entre os diversos pontos da rede, uso limitado de protocolos clínicos que, por sua vez, segundo gestores e profissionais, restringiam o contato entre generalistas e especialistas às referências formais ou contatos informais. Para gestores e profissionais, não havia uso sistemático de contrarreferência, sendo o usuário o principal portador das informações entre os diferentes profissionais. Esses achados revelam uma grande debilidade na região, pois diferentes estudos destacam os instrumentos informativos como mecanismos fundamentais ao processo de fortalecimento da integração dos serviços e coordenação do cuidado 50,51,52.

A deficiência na coordenação do cuidado também era afetada na forma de integração hierárquica (ao invés de rede interdependente) entre a ESF e centros de especialidades. Para gestores e profissionais, não existia contato do médico da USF com especialistas que, por sua vez, não valorizavam o trabalho do generalista. Nesse aspecto, o sucesso na coordenação via atenção primária à saúde depende, também, do prestígio/credibilidade que médicos generalistas desfrutam entre o coletivo de médicos especialistas e com a sociedade 53

Na região, no principal prestador público de serviços especializados, o vínculo trabalhista de médicos era por contrato temporário, gerando rotatividade, e diferentes profissionais concentravam suas atividades em poucos turnos na semana, realizando uma clínica prescritiva (produtora de demandas) e desarticulada da atenção primária à saúde (sem fluxo informacional), além de se comportarem como prestadores autônomos, sem sinergia com a rede. Um gestor municipal descreve tal cenário: “...temos aqui [centro de especialidades] uma miscelânea de profissionais. (...) cada médico quer ter uma conduta, quer colocar sua norma, como ele trabalha na rede particular (...). A rotatividade é grande, existem profissionais que vêm para o SUS para fazer nome" (Ent.8-GM).

Em observação, constatou-se que a organização interna do serviço de especialidades também dificultava uma maior coesão entre prestadores de serviços (médicos especialistas) e demais pontos da rede porque não havia registros eletrônicos das informações que permitissem compartilhamento entre diferentes níveis, e os prontuários clínicos, além de preenchidos manualmente, eram de difícil acesso, mesmo para os profissionais do próprio estabelecimento.

Vale notar, segundo gestores e observações na CIR, que as estratégias locorregionais para superar a insuficiência na oferta, ainda que importantes e fundamentais, contraditoriamente não fortaleciam a integração e corroíam a coordenação, pois não logravam envolver as equipes de saúde da família ou limitavam sua participação ao processo de revisão das guias de solicitação para reclassificação das prioridades. Entre as estratégias para aumentar a oferta, os gestores destacaram a compra de consultas/exames da rede privada e realização de mutirões, particularmente nas especialidades com maior tempo de espera. Ambas as táticas aumentam a oferta e resolvem problemas pontuais de acesso, mas tendem a apresentar resultados transitórios, dado que não modificam o padrão de produção do cuidado, não alteram o núcleo tecnológico do processo de trabalho, não envolvem interação de projetos terapêuticos entre os níveis e, por fim, tornam-se barreiras à coordenação do cuidado.

\section{Considerações finais}

Na região de saúde, pontos de atenção secundária e sistema de apoio atuavam de forma fragmentada da atenção primária à saúde, com fluxos comunicacionais deficientes entre si ou mesmo com ausência informacional entre os profissionais e/ou serviços. Tal questão afetava as tentativas de integração e comprometiam a longitudinalidade do cuidado. A relação entre equipes de saúde da família e especialistas se fazia por meio de precário fluxo burocrático de preenchimento de impressos, mediada por centrais de marcação de consultas. Tais evidências expõem a carência de instrumentos de integração que, por sua vez, obstrui as possibilidades de coordenação entre níveis.

Percebe-se, de modo intrigante, que no processo de regionalização há o esmaecimento da coordenação do cuidado. Isso porque a coordenação pela atenção primária à saúde requer definição do caminhar do usuário ao longo da rede com base na avaliação da equipe de saúde da família, fluxos instituídos e mecanismos de articulação bem estabelecidos entre os níveis. Porém, em redes regionais, os fluxos intermunicipais se dão por mediação administrativa, ou seja, o profissional de atenção primária à saúde se configura como coordenador restrito, quando muito, ao espaço intramunicipal. Sen- 
do assim, quanto mais o município necessitar de outros entes federados para prestação de serviços, mas frágil será a coordenação clínica da atenção primária à saúde, sobretudo quando os mecanismos comunicacionais são precários, as equipes não sabem ou não têm para onde encaminhar os usuários. Nessa lógica, não há como existir coordenação e passa-se a ter somente muitos encaminhamentos.

Essa condução explicita uma rede fracionada, pouco resolutiva e, em certa medida, que não conseguia desobstruir as agendas, pois congestionava os fluxos em todas as direções e, em consequência, reverberava na crônica insuficiência de vagas, no mau uso do recurso público e na insatisfação de gestores, profissionais e usuários.

Destaca-se como ponto forte da pesquisa o desenvolvimento de estudo de caso que investiga a ESF num contexto de região de saúde, ressaltando a posição da ESF nas políticas de integração de serviços numa região de saúde. As investigações acerca da coordenação do cuidado no Brasil, de maneira geral, abordam a relação entre profissionais da atenção primária à saúde e profissionais de outros níveis em cenários intramunicipais. Nesse ínterim, investigar os limites e possibilidades advindas de contextos de redes regionalizadas traz importantes indicativos para formulação ou readequação de políticas de saúde. Salienta-se que não foram encontrados outros estudos de caso sobre o tema na literatura revisada.

Por fim, a opção pela pesquisa qualitativa não é suficiente para análise de todos os elementos de uma rede regionalizada para compreensão da gestão do cuidado integral, requerendo outras abordagens para ampliar o escopo dos dados e, assim, possibilitar uma triangulação de métodos. Sugere-se, também, ampliar o estudo para diferentes regiões de saúde e realizar uma análise comparada entre essas, aumentando a validade externa.

\section{Colaboradores}

A. M. Santos realizou a pesquisa como parte da tese de doutorado, analisou os dados, redigiu e organizou o artigo. L. Giovanella orientou a pesquisa, participou da concepção e revisou o artigo.

\section{Agradecimentos}

Agradecemos aos participantes do estudo. 


\section{Referências}

1. Cecílio LCO. A morte de Ivan Ilitch, de Leon Tols tói: elementos para se pensar as múltiplas dimensões da gestão do cuidado. Interface Comun Saúde Educ 2009; 13 Suppl 1:545-55.

2. Fleury S, Ouverney AM. Gestão de redes: a estratégia de regionalização da política de saúde. Rio de Janeiro: Editora FGV; 2007.

3. Mendes EV. As redes de atenção à saúde. 2a Ed. Brasília: Organização Pan-Americana da Saúde; 2011.

4. Hartz ZMA, Contandriopoulos A-P. Integralidade da atenção e integração de serviços de saúde: desafios para avaliar a implantação de um "sistema sem muros”. Cad Saúde Pública 2004; 20 Suppl 2:S331-6.

5. Machado CV, Lima LD, Viana ALA, Oliveira RG, Iozzi FL, Albuquerque MV, et al. Federalismo e política de saúde: comissões intergovernamentais no Brasil. Rev Saúde Pública 2014; 48:642-50.

6. Viana ALD, Lima LD, Ferreira MP. Condicionantes estruturais da regionalização na saúde: tipologia dos Colegiados de Gestão Regional. Ciênc Saúde Coletiva 2010; 15:2317-26.

7. Campos GWS. Efeitos paradoxais da descentralização no Sistema Único de Saúde do Brasil. In: Fleury S, organizadora. Democracia, descentralização e desenvolvimento: Brasil e Espanha. Rio de Janeiro: Editora FGV; 2006. p. 417-42.

8. Vianna RP, Lima LD. Colegiados de gestão regional no estado do Rio de Janeiro: atores, estratégias e negociação intergovernamental. Physis (Rio J.) 2013; 23:1025-49.

9. Viegas SMF, Penna CMM. O SUS é universal, mas vivemos de cotas. Ciênc Saúde Coletiva 2013; 18:181-90.

10. Spedo SM, Pinto NRS, Tanaka OY. O difícil acesso a serviços de média complexidade no SUS: o caso da cidade de São Paulo, Brasil. Physis (Rio J.) 2010; 20:953-72

11. Ianni AMZ, Monteiro PHN, Alves OSF, Morais MLS, Barboza R. Metrópole e região: dilemas da pactuação da saúde. O caso da região metropolitana da Baixada Santista, São Paulo, Brasil. Cad Saúde Pública 2012; 28:925-34.

12. Viana ALD, Ibañez N, Elias PEM, Lima LD, Albuquerque MV, Iozzi FL. Novas perspectivas para a regionalização da saúde. São Paulo Perspect 2008; 22:92-106.

13. Vieira-da-Silva LM, Hartz ZMA, Chaves SCL, Silva GAP, Paim JS. Análise da implantação da gestão descentralizada em saúde: estudo comparado de cinco casos na Bahia, Brasil. Cad Saúde Pública 2007; 23:355-70.

14. Giovanella L, Mendonça MHM, Almeida PF, Escorel S, Senna MCM, Fausto MCR, et al. Saúde da família: limites e possibilidades para uma abordagem integral de atenção primária à saúde no Brasil. Ciênc Saúde Coletiva 2009; 14:783-94.

15. Escorel S, Giovanella L, Mendonça MHM, Senna MCM. O Programa de Saúde da Família e a construção de um novo modelo para a Atenção Básica no Brasil. Rev Panam Salud Pública 2007; 21:164-76
16. Kushnir R, Chorny AH. Redes de atenção à saúde: contextualizando o debate. Ciênc Saúde Coletiva 2010; 15:2307-16.

17. Lopes CMN. Regionalização em saúde: o caso de uma microrregião no Ceará (1998-2002 e 20072009) [Tese de Doutorado]. Salvador: Instituto de Saúde Coletiva, Universidade Federal da Bahia; 2010.

18. Coelho APS. O público e o privado na regionalização da saúde: processo decisório e condução da política no estado do Espírito Santo [Dissertação de Mestrado]. Rio de Janeiro: Escola Nacional de Saúde Pública Sergio Arouca, Fundação Oswaldo Cruz; 2011.

19. Santos AM, Giovanella L. Governança regional: estratégias e disputas para gestão em saúde. Rev Saúde Pública 2014; 48:622-31.

20. Pereira AMM. Dilemas federativos e regionalização da saúde: o papel do gestor estadual do SUS em Minas Gerais [Dissertação de Mestrado]. Rio de Janeiro: Escola Nacional de Saúde Pública Sergio Arouca, Fundação Oswaldo Cruz; 2009.

21. Neves LA, Ribeiro JM. Consórcios de saúde: estudo de caso exitoso. Cad Saúde Pública 2006; 22: 2207-17.

22. Lima LD, Machado CV, O’Dwyer G, Baptista TWT, Andrade CLT, Konder MT. Interdependência federativa na política de saúde: a implementação das Unidades de Pronto Atendimento no estado do Rio de Janeiro, Brasil. Ciênc Saúde Coletiva 2015; 20:595-606.

23. Greß S, Baan CA, Calnan M, Dedeu T, Groenwegen $\mathrm{P}$, Howson $\mathrm{H}$, et al. Co-ordination and management of chronic conditions in Europe: the role of primary care - position paper of the European Forum for Primary Care. Qual Prim Care 2009; 17:75-86.

24. Saltman RB, Rico A, Boerma WGW. Atenção Primária conduzindo as redes de atenção à saúde: reforma organizacional na atenção primária europeia. Berkshire: Open University Press; 2006.

25. Wadmann S, Strandberg-Larsen M, Vrangbek K. Coordination between primary and secondary healthcare in Denmark and Sweden. Int J Integr Care 2009; 9:1-14.

26. Vázquez ML, Vargas I, Unger J, Mogollón, Silva MRF, Paepe P. Integrated health care network in Latin America: toward a conceptual framework for analysis. Rev Panam Salud Pública 2009; 26:360-7.

27. Armitage GD, Suter E, Oelke ND, Adair CE. Health systems integration: state of the evidence. Int J Integr Care 2009; 9:1-11.

28. Yin RK. Estudo de caso: planejamento e métodos. 3a Ed. Porto Alegre: Bookman; 2005.

29. Vilasbôas ALQ, Paim JS. Práticas de planejamento e implementação de políticas no âmbito municipal. Cad Saúde Pública 2008; 24:1239-50.

30. Cunha ABO, Vieira-da-Silva LM. Acessibilidade aos serviços de saúde em um município do Estado da Bahia, Brasil, em gestão plena do sistema. Cad Saúde Pública 2010; 26:725-37. 
31. Ministério da Saúde. Decreto no 7.508, de 28 de junho de 2011. Regulamenta a Lei no 8.080, de 19 de setembro de 1990, para dispor sobre a organização do SUS, o planejamento da saúde, a assistência à saúde e a articulação interfederativa, e dá outras providências. Diário Oficial da União 2011; 29 jun.

32. Alexandre NMC, Coluci MZO. Validade de conteúdo nos processos de construção e adaptação de instrumentos de medida. Ciênc Saúde Coletiva 2011; 16:3061-8.

33. Lynn MR. Determination and quantification of content validity. Nurs Res 1986; 35:382-5.

34. Starfield B. Atenção primária: equilíbrio entre necessidades de saúde, serviços e tecnologia. Brasília: Organização das Nações Unidas para a Educação, a Ciência e a Cultura/Ministério da Saúde; 2002.

35. Elias PE, Ferreira CW, Alves MCG, Cohn A, Kishima V, Escrivão Jr. A, et al. Atenção básica em saúde: comparação entre PSF e UBS por extrato de exclusão social no município de São Paulo. Ciênc Saúde Coletiva 2006; 11:633-41.

36. Ibañez N, Rocha JSY, Castro PC, Ribeiro MCSA, Forster AC, Novaes MHD, et al. Avaliação do desempenho da atenção básica no Estado de São Paulo. Ciênc Saúde Coletiva 2006; 11:683-703.

37. Macinko J, Almeida C, Oliveira ES. Avaliação das características organizacionais dos serviços de atenção básica em Petrópolis: teste de uma metodologia. Saúde Debate 2003; 27:243-56.

38. Kringos D, Boerma W, Bourgueil Y, Cartier T, Dedeu T, Hasvold T, et al. The strength of primary care in Europe: an international comparative study. $\mathrm{Br} \mathrm{J}$ Gen Pract 2013; 63:e742-50.

39. Hernández T, Gómez YO. La migración de médicos en Venezuela. Rev Panam Salud Pública 2011; 30:177-81.

40. Kringos DS, Boerma WGW, van der Zee J, Groenewegen PP. The contribution of primary care to health care system performance in Europe. In: Kringos DS, editor. The strength of primary care in Europe. Utrecht: NIVEL/University of Utrecht; 2012. p. 217-37.

41. Gérvas J, Borris J. El debate profesional acerca de la escasez de médicos. Rev Esp Salud Pública 2008; 82:627-35.

42. Mendonça MHM, Martins MIC, Giovanella L, Escorel S. Desafios para gestão do trabalho a partir de experiências exitosas de expansão da Estratégia de Saúde da Família. Ciênc Saúde Coletiva 2010; 15:2355-65.
43. Ney MS, Rodrigues PHA. Fatores críticos para fixação do médico na Estratégia Saúde da Família. Physis (Rio J.) 2012; 22:1293-311.

44. Geneau R, Lehoux P, Pineault R, Lamarche PA. Primary care practice à la carte among GPs: using organizational diversity to increase job satisfaction. Fam Pract 2007; 24:138-44.

45. Bousquat A, Alves MCGP, Elias PE. Utilização do Programa Saúde da Família em regiões metropolitanas: abordagem metodológica. Rev Saúde Pública 2008 ; 42:903-6.

46. Fernandes LC, Bertoldi AD, Barros AJD. Utilização dos serviços de saúde pela população coberta pela Estratégia de Saúde da Família. Rev Saúde Pública 2009; 43:595-603.

47. Ministério da Saúde. Diretrizes para a programação pactuada e integrada da assistência à saúde. Brasília: Ministério da Saúde; 2006.

48. Venancio SI, Nascimento PR, Rosa TE, Morais MLS, Martins PN, Voloschko A. Referenciamento regional em saúde: estudo comparado de cinco casos no Estado de São Paulo, Brasil. Ciênc Saúde Coletiva 2011; 16:3951-64.

49. Pires MRG, Göttems LB, Martins CMF, Guilhem D, Alves ED. Oferta e demanda por média complexidade/SUS: relação com atenção básica. Ciênc Saúde Coletiva 2010; 15:1009-19.

50. Almeida PF, Giovanella L, Mendonça MHM, Escorel S. Desafios à coordenação dos cuidados em saúde: estratégias de integração entre níveis assistenciais em grandes centros urbanos. Cad Saúde Pública 2010; 26:286-98.

51. Almeida PF, Gérvas J, Frieire J, Giovanella L. Estratégias de integração entre atenção primária à saúde e atenção especializada: paralelos entre Brasil e Espanha. Saúde Debate 2013; 37:400-15.

52. Vázquez ML, Vargas I, Nuño R, Toro N. Organizaciones sanitarias integradas y otros ejemplos de colaboración entre proveedores. Informe SESPAS 2012. Gac Sanit 2012; 26:94-101.

53. Gérvas J, Starfield B, Minué S, Violan C. Algunas causas (y soluciones) de la pérdida de prestigio de la medicina general/de familia. Contra el descrédito del héroe. Aten Primaria 2007; 39:615-8. 


\section{Abstract}

This study analyzed management of comprehensive care in a health district in Bahia State, Brazil, at the political, institutional, organizational, and healthcare practice levels and the challenges for establishing coordinated care between municipalities. The information sources were semi-structured interviews with administrators, focal groups with healthcare professionals and users, institutional documents, and observations. A comprehensive and critical analysis was produced with dialectical hermeneutics as the reference. The results show that the Inter-Administrators Regional Commission was the main regional governance strategy. There is a fragmentation between various points and lack of communications linkage in the network. Private interests and partisan political interference overlook the formally agreed-upon flows and create parallel circuits, turning the right to health into currency for trading favors. Such issues hinder coordination of comprehensive care in the inter-municipal network

Regional Health Planning; Primary Health Care;

Health Management; Integrality in Health;

Clinical Governance

\section{Resumen}

Se analizó la conformación de la gestión del cuidado integral en los niveles político-institucional, organizativo y, en la práctica, identificando los desafios para la constitución de cuidados coordinados entre municipios y los dispositivos para la integración y regulación asistencial. Se trata de un estudio de caso, con un enfoque cualitativo, en la región sanitaria de Vitória da Conquista, Bahía, Brasil, que abarca a 19 municipios. Las fuentes de información fueron entrevistas semiestructuradas con gestores, grupos focales con gestores, profesionales y usuarios, documentos institucionales y observaciones. Los resultados indicaron la insuficiencia de médicos para las acciones y servicios en la Estrategia Salud de la Familia, así como en los servicios especializados, limitando el acceso oportuno. Había fragmentación entre los diferentes puntos y desarticulación comunicativa en la red. Intereses privados e interferencias clientelistas creaban circuitos paralelos, transformando el derecho a la salud en un intercambio de favores. Tales cuestiones obstruyen las posibilidades de coordinación del cuidado en la red intermunicipal.

Regionalización; Atención Primaria de Salud; Gestión en Salud; Integralidad en Salud; Gestión Clínica
Recebido em 18/Nov/2014

Versão final reapresentada em 17/Jul/2015 Aprovado em 23/Jul/2015 